\title{
Easy Calibration for Para-catadioptric-like Camera
}

\author{
Yihong $\mathrm{Wu}^{1,2}$, Youfu $\mathrm{Li}^{1}$, and Zhanyi $\mathrm{Hu}^{2}$ \\ ${ }^{1}$ Dept. of Manufacturing Engg and Engg Management \\ ${ }^{2}$ National Laboratory of Pattern Recognition \\ City University of Hong Kong \\ Hong Kong, P.R. China \\ \{yhwu, meyfli\}@cityu.edu.hk \\ Institute of Automation, Chinese Academy of Sciences \\ Beijing, P.R. China \\ \{yhwu, huzy\}@nlpr.ia.ac.cn
}

\begin{abstract}
For omnidirectional cameras, most of the previous calibration methods from lines use conic fitting. This paper presents a calibration method for para-catadioptric-like cameras from lines without conic fitting under a single view. We establish equations on the five camera intrinsic parameters. These equations are linear for the focal lengths and skew factor once the principal point is known. The principal point can be approximated well by the center of the imaged mirror contour in practice or can be accurately estimated by quadric equations. After obtaining the principal point, we propose an algorithm to calibrate the focal lengths and skew factor. The algorithm needs neither prior structure knowledge nor conic fitting and is linear, which make it easy to implement. Other omnidirectional cameras can also use this presented work if high accuracy is not required. Experiments demonstrate the efficiency of the proposed algorithm.
\end{abstract}

Index Terms - Omnidirectional camera, camera calibration.

\section{INTRODUCTION}

Omnidirectional cameras have a large field of view offering great benefit to three-dimensional modeling of wide environment, robot navigation, and visual surveillance. Camera calibration is required in these fields of vision applications.

There have been studies on omnidirectional camera calibration in recent years as follows:

- Some of the methods use known information of scene points to calibrate cameras under a single view [1, 2, 3 , 4]. For example, the focal length and the position of relaxing paraboloid catadioptric cameras were calibrated from known three-dimensional (3D) space points in [1]. In [2], the principal point of some omnidirectional cameras with single axis was computed accurately from one-dimensional (1D) or two-dimensional (2D) space points. Reference [3] calibrated a camera system, where each sub-camera was calibrated from known 3D points. Using known 2D or 3D space points, [4] provided a calibration method for a highly generic kind of omnidirectional cameras including both central and non-central cameras.

- Another kind of the methods is from correspondences between multiple views without requiring any scene information [2, 5, 6, 7, 8]. Specific camera motions were used to calibrate generic cameras in [5].
- Lines, projected as conics on image plane, are also considered for omnidirectional camera calibration under a single view [9, 10, 11, 12, 13]. Geyer and Daniilidis calibrated a paraboloid catadioptric camera by fitting image points from parallel lines as circles [9]. Later, they improved their method, of which no parallelity was needed [10]. In [11, 12, 13], fitting line images as conics was made when calibrating central catadioptric cameras.

In this work, also from lines but without conic fitting we provide a calibration method for para-catadioptric-like cameras. The para-catadioptric-like cameras are defined as paraboloid catadioptric cameras, or omnidirectional cameras that can be described by a sphere model [14] with mirror parameter approximately being 1 . For this kind of cameras, we find that the two focal lengths and the skew factor can be linearly calculated by image points from lines once the principal point is known.

Advantages of this method include:

(i) Only image points from lines under a single view are used and no space relative structure is needed.

(ii) One line is enough to calibrate the two focal lengths and the skew factor. More lines can be used to increase stability of the method.

(iii) No fitting of image points to conics is required and just taking three image points from a same space line can directly give an equation on the intrinsic parameters. This is a difference compared with the previous methods using lines. As it is known for the omnidirectional cameras only small portion of conics of line images is visible such that the usual conic fitting method does not work well.

(iv) The method is linear.

These advantages make the calibration method easy to implement.

Due to its easy implementation, this method can also be considered by other omnidirectional cameras to obtain a rough intrinsic parameter estimation if high accuracy is not required.

Before introducing our work, we would like to say the center of the imaged mirror contour can be as the principal point well in practice, or the principal point can be accurately computed by a quasi-linear method based on quadric equations. In this paper, we use the center of the imaged mirror contour as the principal point. The quasi-linear method will be reported in future. 
The remainder of this paper is organized as follows: Section 2 introduces the camera models. Section 3 reports the calibration method and then experiments are followed in Section 4. Conclusion is given in Section 5.

\section{CAMERA MODELS}

\section{A. Perspective Camera}

Under a perspective camera model [15], a space point $\mathbf{M}$ is projected to an image point $\mathbf{m}$ by:

$$
\mathbf{m} \approx \mathbf{K}(\mathbf{R}, \mathbf{t}) \mathbf{M}=\mathbf{K}(\mathbf{R} \hat{\mathbf{M}}+\mathbf{t}),
$$

where $\approx$ denotes the equality up to a scalar, $\mathbf{M}=\left(\hat{\mathbf{M}}^{T} 1\right)^{T}$ is the homogeneous coordinates of the space point under the world coordinate system, $(\mathbf{R}, \mathbf{t})$ are a $3 \times 3$ rotation matrix and a 3 -vector of translation that are the camera motion parameters. And

$$
\mathbf{K}=\left(\begin{array}{ccc}
f_{u} & s & u_{0} \\
0 & f_{v} & v_{0} \\
0 & 0 & 1
\end{array}\right)
$$

is the matrix of camera intrinsic parameters, $f_{u}$ and $f_{v}$ are the focal lengths, $s$ the skew, and $\left(u_{0}, v_{0}\right)$ the principal point.

\section{B. Catadioptric Camera}

A central catadioptric camera model is an equivalent spherical projection defined by Geyer and Daniilidis [14], as shown in Fig. 1. A space point $\mathbf{M}$ is projected to a point $\mathbf{X}$ on the viewing sphere through the sphere center $\mathbf{O}$, and then projected to $\mathbf{m}$ on the image plane through the camera viewpoint $\mathbf{O}_{c}$. The camera optical axis is the line $\mathbf{O O}_{c}$. The process can be described as:

$$
\mathbf{m} \approx \mathbf{K}\left(\frac{\mathbf{R} \hat{\mathbf{M}}+\mathbf{t}}{\|\mathbf{R} \hat{\mathbf{M}}+\mathbf{t}\|}+\left(\begin{array}{lll}
0 & 0 & l
\end{array}\right)^{T}\right),
$$

where $\left(\hat{\mathbf{M}}^{T} 1\right)^{T}, \mathbf{K}$, and $(\mathbf{R}, \mathbf{t})$ are as in (1), \|\| denotes the norm of vector in it, and $l$ is the mirror parameter that is the distance from $\mathbf{O}$ to $\mathbf{O}_{c}$. The mirror is a paraboloid if $l=1$, an ellipsoid or hyperboloid if $0<l<1$, and a plane if $l=0$.

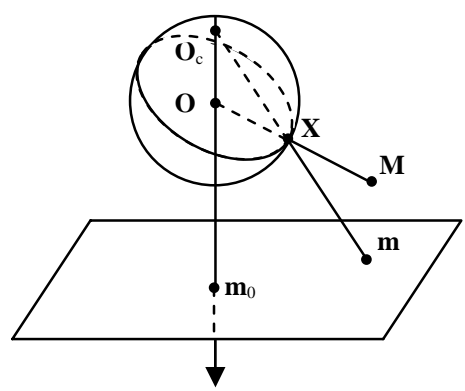

Fig. 1 Imaging process under central catadioptric model
The images of catadioptric cameras have distortions. A straight line in space is projected into a conic on the image plane called as a line image.

\section{Para-catadioptric-like Camera}

The mirror parameter $l$ for the paraboloid catadioptric camera is 1 . Other some omnidirectional cameras can also be approximate to the sphere model with the mirror parameter $l$ as 1 . This kind of omnidirectional cameras and the paraboloid catadioptric cameras are defined as para-catadioptric-like cameras.

For example, a perspective camera combined with a FCE8 fisheye lens and a perspective camera combined with a hyperboloid mirror designed by the Center for Machine Perception of the Czech technical University can be regarded as a para-catadioptric-like camera if high accuracy is not required. This approximation performed can simplify some complex vision task such as calibration, 3D reconstruction and et al.

\section{CALIBRATION FOR PARA-CATADIOPTRIC-LIKE CAMERA}

\section{A. Shift from Catadioptric Image to Perspective Image}

In this subsection, we consider all the catadioptric cameras defined by (2).

Denote the homogeneous principal point $\left(u_{0}, v_{0}, 1\right)^{T}$ as $\mathbf{m}_{0}$. Then (2) is equivalent to:

$$
s_{m} \mathbf{m}-l \mathbf{m}_{0}=\mathbf{K} \frac{\mathbf{R} \hat{\mathbf{M}}+\mathbf{t}}{\|\mathbf{R} \hat{\mathbf{M}}+\mathbf{t}\|} \approx \mathbf{K}(\mathbf{R} \hat{\mathbf{M}}+\mathbf{t}) .
$$

where $s_{m}$ is a nonzero scalar related to $\mathbf{m}$. Comparing (3) with (1), we find that $s_{m} \mathbf{m}-l \mathbf{m}_{0}$ just can be as an image point of $\mathbf{M}$ under some perspective camera. And $\mathbf{K}^{-1}\left(s_{m} \mathbf{m}-l \mathbf{m}_{0}\right)$ just is the corresponding point of $\mathbf{m}$ on the viewing sphere.

$l$ is the mirror parameter that may be known from manufacturer but $s_{m}$ cannot be obtained easily. This is because $s_{m}$ is related to the camera intrinsic parameters as follows.

By (3), we have:

$$
\mathbf{K}^{-1}\left(s_{m} \mathbf{m}-l \mathbf{m}_{0}\right)=\frac{\mathbf{R} \hat{\mathbf{M}}+\mathbf{t}}{\|\mathbf{R} \hat{\mathbf{M}}+\mathbf{t}\|} .
$$

Doing inner product from each side of this equation yields:

$$
\left(s_{m} \mathbf{m}-l \mathbf{m}_{0}\right)^{T} \mathbf{K}^{-T} \mathbf{K}^{-1}\left(s_{m} \mathbf{m}-l \mathbf{m}_{0}\right)=1 .
$$

The result is a quadric equation on $s_{m}$. Solving for $s_{m}$ and simplifying the result by $\mathbf{K}^{-1} \mathbf{m}_{0}=\left(\begin{array}{lll}0 & 0 & 1\end{array}\right)^{T}$, $\mathbf{m}_{0}^{T} \mathbf{K}^{-T} \mathbf{K}^{-1} \mathbf{m}_{0}=1, \mathbf{m}^{T} \mathbf{K}^{-T} \mathbf{K}^{-1} \mathbf{m}_{0}=1$ give the two solutions: 


$$
s_{m}=\frac{l \pm \sqrt{l^{2}+\left(1-l^{2}\right) \mathbf{m}^{T} \mathbf{K}^{-T} \mathbf{K}^{-1} \mathbf{m}}}{\mathbf{m}^{T} \mathbf{K}^{-T} \mathbf{K}^{-1} \mathbf{m}} .
$$

The expression in (4) is a function on $l$ and $\mathbf{K}$. It follows that if we do not know $l$ and $\mathbf{K}$, it is not possible to transform the catadioptric image into the perspective image or into the viewing sphere by $\mathbf{K}^{-1}\left(s_{m} \mathbf{m}-l \mathbf{m}_{0}\right)$.

\section{B. Calibration Equation for Para-catadioptric-like Camera}

Hereafter, all the considered cameras are paracatadioptric-like cameras. For para-catadioptric-like cameras, $l=1$.

Substituting $l=1$ into (4) gives 0 and:

$$
s_{m}=\frac{2}{\mathbf{m}^{T} \mathbf{K}^{-T} \mathbf{K}^{-1} \mathbf{m}} .
$$

Since the zero solution is not the true solution, we only consider (5). Let $(u, v)$ be the first two coordinates of $\mathbf{m}-\mathbf{m}_{0}, \mathbf{K}_{0}$ be the resulting $\mathbf{K}$ after substituting $u_{0}=0$ and $v_{0}=0$. The matrix $\mathbf{K}_{0}^{-T} \mathbf{K}_{0}^{-1}$ is in this form:

$$
\left(\begin{array}{ccc}
k_{1}, & k_{3} / 2, & 0 \\
k_{3} / 2, & k_{2}, & 0 \\
0, & 0, & 1
\end{array}\right) .
$$

Then, (5) can be changed into:

$$
s_{m}=\frac{2}{1+k_{1} u^{2}+k_{2} v^{2}+k_{3} u v} .
$$

For three image points $\mathbf{m}_{1}, \mathbf{m}_{2}, \mathbf{m}_{3}$ on a line image, their transformed perspective image points $s_{m_{1}} \mathbf{m}_{1}-l \mathbf{m}_{0}=s_{m_{1}} \mathbf{m}_{1}-\mathbf{m}_{0}, s_{m_{2}} \mathbf{m}_{2}-l \mathbf{m}_{0}=s_{m_{2}} \mathbf{m}_{2}-\mathbf{m}_{0}$, $s_{m_{3}} \mathbf{m}_{3}-l \mathbf{m}_{0}=s_{m_{3}} \mathbf{m}_{3}-\mathbf{m}_{0}$ will be collinear. Thus the determinant of these three transformed points is zero. By expanding this determinant and diving the result by $s_{m_{1}} s_{m_{2}} s_{m_{3}}$, we have:

$$
\begin{aligned}
& \frac{1}{s_{m_{3}}}\left[\mathbf{m}_{1} \mathbf{m}_{2} \mathbf{m}_{0}\right]-\frac{1}{s_{m_{2}}}\left[\mathbf{m}_{1} \mathbf{m}_{3} \mathbf{m}_{0}\right]+\frac{1}{s_{m_{1}}}\left[\mathbf{m}_{2} \mathbf{m}_{3} \mathbf{m}_{0}\right] \\
& -\left[\mathbf{m}_{1} \mathbf{m}_{2} \mathbf{m}_{3}\right]=0,
\end{aligned}
$$

where the bracket [ ] denotes determinant of vectors in it. Substituting each $s_{m_{i}}$ by the corresponding equation (7) into

(8) yields:

$$
\begin{aligned}
& \left(u_{3}^{2}\left[\mathbf{m}_{1} \mathbf{m}_{2} \mathbf{m}_{0}\right]-u_{2}^{2}\left[\mathbf{m}_{1} \mathbf{m}_{3} \mathbf{m}_{0}\right]+u_{1}^{2}\left[\mathbf{m}_{2} \mathbf{m}_{3} \mathbf{m}_{0}\right]\right) k_{1} \\
& +\left(v_{3}^{2}\left[\mathbf{m}_{1} \mathbf{m}_{2} \mathbf{m}_{0}\right]-v_{2}^{2}\left[\mathbf{m}_{1} \mathbf{m}_{3} \mathbf{m}_{0}\right]+v_{1}^{2}\left[\mathbf{m}_{2} \mathbf{m}_{3} \mathbf{m}_{0}\right]\right) k_{2} \\
& +\left(u_{3} v_{3}\left[\mathbf{m}_{1} \mathbf{m}_{2} \mathbf{m}_{0}\right]-u_{2} v_{2}\left[\mathbf{m}_{1} \mathbf{m}_{3} \mathbf{m}_{0}\right]+u_{1} v_{1}\left[\mathbf{m}_{2} \mathbf{m}_{3} \mathbf{m}_{0}\right]\right) k_{3} \\
& -\left[\mathbf{m}_{1} \mathbf{m}_{2} \mathbf{m}_{3}\right]=0,
\end{aligned}
$$

where $\left(u_{i}, v_{i}\right)$ are the first two coordinates of $\mathbf{m}_{i}-\mathbf{m}_{0}$. It is clear that (9) is a linear equation on $k_{1}, k_{2}$, and $k_{3}$.

By different equations (9), we can eliminate $k_{1}, k_{2}, k_{3}$ and the results are quadric equations on $\mathbf{m}_{0}$. The quadric equations can be used to accurately compute $\mathbf{m}_{0}$ by a quasi- linear method. Similarly, conic fitting is not needed for this quasi-linear method. This will be given in future. Here, we use the center of the imaged mirror contour as $\mathbf{m}_{0}$ (see the analysis in the simulations of the experiments).

\section{Implementation}

Among equations (9) from different triples of image points on a same line image, there are three equations to be independent. Thus, in theory one line image is enough to solve the parameters $k_{1}, k_{2}, k_{3}$. More line images can be used to increase calculation stability. Assume there are $N$ line images. The implementation of the proposed calibration method is given as:

Step 1. Along each line image, extract its image points and put the extraction into a set. So, $N$ number of sets are obtained and denoted as $S T_{i}, i=1, \ldots, N$.

Step 2. For each $S T_{i}$, let $P_{1}, P_{2}, P_{3}$ be the first, the middle, and the last points in it. Then, the set of the remaining points in $S T_{i}$ is denoted by $R P$. Combine $\left(P_{1}, P_{2}\right)$ and each of points in $R P$ outside the neighborhood of $P_{1}, P_{2}$ to form different triples. Repeat the process for $\left(P_{1}, P_{3}\right)$ and repeat the process for $\left(P_{2}, P_{3}\right)$. Put all of the triples obtained into a set $G$.

Step 3. For each triple in $G$, establish the corresponding linear equation (9). Then, solve all of the obtained equations by SVD method for $k_{1}, k_{2}, k_{3}$.

Step 4. Do optimization for $k_{1}, k_{2}, k_{3}$, where the cost function is the sum of the residual squares of the equations in Step 3.

Step 5. Applying Proposition 1 in [16] to (6), we obtain the final calibrated parameters as:

$$
f_{u}=\frac{1}{\sqrt{k_{1}}}, f_{v}=\sqrt{\frac{k_{1}}{k_{1} k_{2}-\frac{k_{3}^{2}}{4}}}, s=-\frac{k_{3}}{2 k_{1}} f_{v} .
$$

Remark: Step 4 is optional. Because image points from lines are abundant, we can establish a great lot of equations on the camera parameters in Step 3. Furthermore, SVD method self is a least-square optimization method. Therefore usually without Step 4, satisfactory estimations can still be obtained and simultaneously the implementation is fast.

\section{EXPERIMENTS}

Many experiments performed validate the established calibration method. One sample in simulation and the results from two real images are reported as follows. Due to little improvement by Step 4 in the proposed algorithm, the 
following shown results were computed without using this step.

\section{A. Simulations}

The simulated camera intrinsic parameters were:

$$
\mathbf{K}=\left(\begin{array}{ccc}
610 & 0.8 & 500 \\
0 & 600 & 350 \\
0 & 0 & 1
\end{array}\right)
$$

where $(500,350)$ is the principal point $\mathbf{m}_{0}$ assumed to be known, 0.8 is the skew factor $s, 610$ and 600 are the two focal lengths $f_{u}$ and $f_{v}$. Six space lines, each consisting of 201 points, were projected to the simulated image plane through a paraboloid catadioptric camera. The result is shown in Fig. 2 and its size is $800 \times 800$ pixels.

Gaussian noise with mean 0 and standard deviation ranging from 0 to 3 pixels was directly added to the principal point and each of the image points. Then, the proposed calibration method was applied. At each noise level, we performed 100 independent runs and the averaged results are shown in TABLE I. The values have higher accuracies. The variances of the values at each noise level were also calculated and are shown in Fig. 3. We can see that the variances are almost linear with noise increasing and $f_{u}, f_{v}$ are more stable to noise.

The same noise level was added to both the principal point and the image points in the above simulations. In order to test stability of our method to the noise of only the principal point, at the fixed noise level 1 pixel to the image points, the varying Gaussian noise as before was directly added to $\mathbf{m}_{0}$. Then, the averages and the standard deviations of the estimated intrinsic parameters were calculated and are shown in Fig. 4 and Fig. 5 respectively. The results illustrate the proposed method is quite stable to the deviations of the principal point. Therefore in practice, we can use the center of the imaged mirror contour as $\mathbf{m}_{0}$.
TABLE I

ESTIMATED INTRINSIC PARAMETERS VS. NOISE

\begin{tabular}{|l|l|l|l|}
\hline Noise(pixel) & $f_{u}$ & $f_{v}$ & $s$ \\
\hline 0 & 610.00 & 600.00 & 0.80 \\
\hline 0.3 & 609.78 & 600.05 & 1.15 \\
\hline 0.6 & 610.04 & 599.82 & 1.08 \\
\hline 0.9 & 610.52 & 600.08 & 1.17 \\
\hline 1.2 & 610.35 & 599.73 & 0.35 \\
\hline 1.5 & 609.58 & 600.21 & 2.53 \\
\hline 1.8 & 610.60 & 600.22 & 1.05 \\
\hline 2.1 & 610.28 & 599.82 & 1.19 \\
\hline 2.4 & 611.18 & 599.98 & -0.03 \\
\hline 2.7 & 609.58 & 600.36 & 1.53 \\
\hline 3 & 610.99 & 600.36 & 0.30 \\
\hline
\end{tabular}

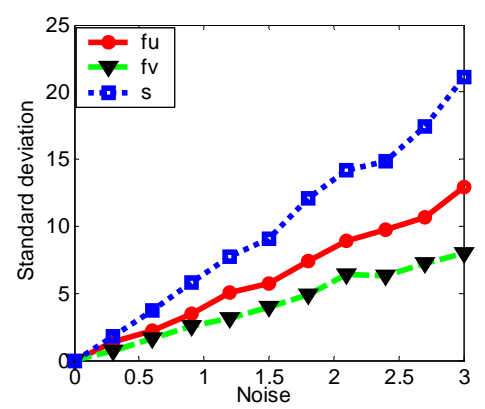

Fig. 3 Standard deviations of the estimated intrinsic parameters vs. noise

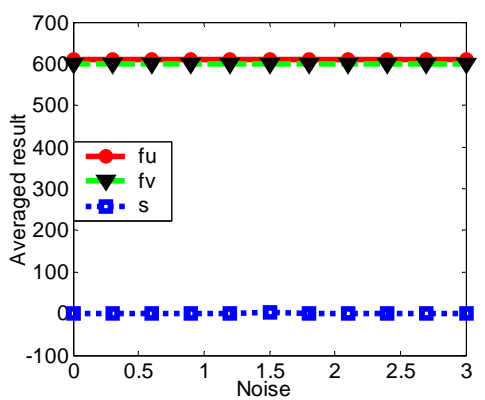

Fig. 4 Estimated intrinsic parameters vs. noise of $\mathbf{m}_{0}$

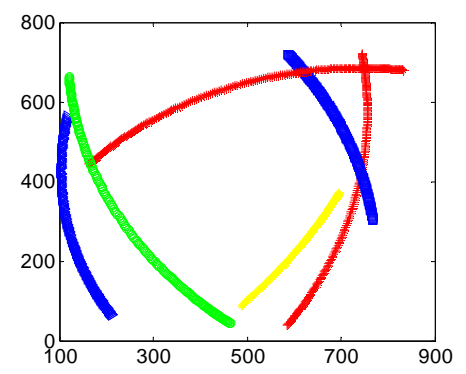

Fig. 2 A simulated para-catadioptric image

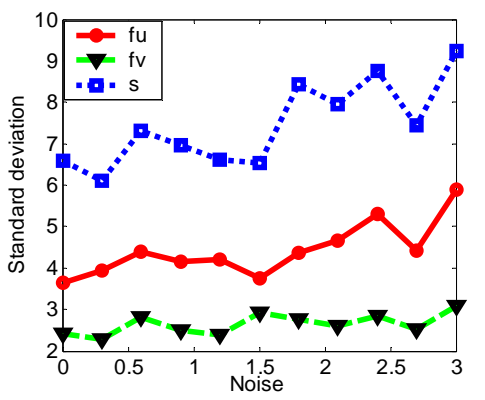

Fig. 5 Standard deviations of the estimated intrinsic parameters vs. noise of $\mathbf{m}_{0}$ 


\section{B. Experiments with Real Data}

The center of the imaged mirror contour was used as $\mathbf{m}_{0}$.

A used real image, captured by a NIKON COOLPIX990 with a FC-E8 fisheye lens, is shown in (a) of Fig. 6. Its size is $512 \times 384$ pixels. Canny edge detector was used to extract the line images and then sample points on each line image were collected by 10-pixel step. From these sample points, the proposed method was applied and the calibrated result is:

$$
f_{u}=172.68, f_{v}=171.34, s=-8.80 \text {. }
$$

In order to verify the estimations, we transformed the original distorted image by these estimated parameters. The result of some part is shown in (b) of Fig. 6. We only show some part of the transformed image is because the transformed image is very large due to the large field of view of the camera. From (b) of Fig. 6, we can see that the distorted lines become straight.

An image of the same size $512 \times 384$ pixels captured by the same camera with a hyperboloid mirror designed by the Center for Machine Perception, Czech technical University is shown in (a) of Fig. 7. The mirror parameter $l$ is 0.966 that is close to 1 . We approximately regarded it as 1 . Applying the proposed method to this image, we obtain the estimated camera parameters as:

$$
f_{u}=141.42, f_{v}=143.70, s=-4.41 .
$$

By these values, the transformation of some part of the original distorted image is shown in (b) of Fig. 7. Similarly, the result demonstrates that those heavy distorted lines become straight.

The above simulations and experiments on real data validate the proposed calibration method and show its efficiency.

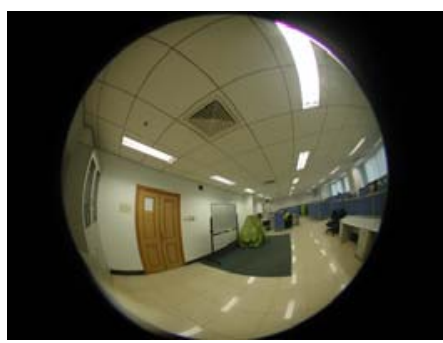

(a)

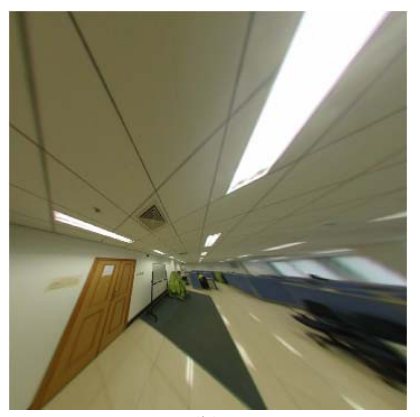

(b)

Fig. 6 (a) A real image from a fisheye lens; (b) Transformation of (a) by the estimated intrinsic parameters

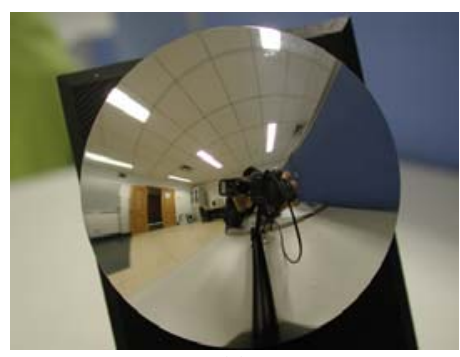

(a)

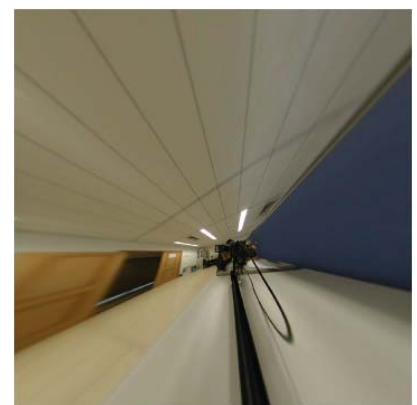

(b)

Fig. 7 (a) A real image from a catadioptric lens;

(b) Transformation of (a) by the estimated intrinsic parameters

\section{CONCLUSION}

A calibration method for para-catadioptric-like cameras from lines under a single view has been presented, where no any prior structure knowledge is needed, no conic fitting is needed, no complex mathematics is needed, and the implementation is linear. These advantages make this method easy to implement. Other omnidirectional cameras can also use this presented work to obtain a rough estimation of intrinsic parameters if high accuracy is not required. At present, we use the center of the imaged mirror contour as the principal point. In future, accurately computation for the principal point by quadric equations will be reported through a quasi-linear algorithm.

\section{ACKNOWLEDGMENT}

This work was supported by a grant from the Research Grants Council of Hong Kong [Project No. CityU1206/04E] and supported by the National Natural Science Foundation of China under grant No. 60475009.

\section{REFERENCES}

[1] D.G. Aliaga, “Accurate catadioptric calibration for real-time pose estimation in room-size environments," The 8th International Conference on Computer Vision, 2001, pp. 127-134.

[2] Y. Wu and Z. Hu, "Geometric Invariants and applications under catadioptric camera model," The 10th International Conference on Computer Vision, 2005, pp. 1547-1554. 
[3] N.D. Jankovic and M.D. Naish, "Calibrating an active omnidirectional vision system,” IEEE/RSJ International Conference on Intelligent Robots and Systems, 2005, pp. 1355-1360.

[4] P. Sturm and S. Ramalingam, "A generic concept for camera calibration,” The 8th European Conference on Computer Vision, 2004, pp. 1-13.

[5] S. Ramalingam, P. Sturm, and S.K. Lodha, "Towards generic selfcalibration of central cameras," The 6th Workshop on Omnidirectional Vision, Camera Networks and Non-classical Cameras, in conjunction with ICCV 2005, pp. 20-27.

[6] B. Micusik and T. Pajdla, "Para-catadioptric camera auto-calibration from epipolar Geometry,” The 6th Asian Conference on Computer Vision, 2004, pp. 748-753.

[7] B. Micusik and T. Pajdla, "Autocalibration \& 3D reconstruction with noncentral catadioptric cameras,” IEEE Conference on Computer Vision and Pattern Recognition, 2004, pp. 58-65.

[8] S.B. Kang, “Catadioptric self-calibration,” IEEE Conference on Computer Vision and Pattern Recognition, 2000, pp. 201-207.

[9] C. Geyer and K. Daniilidis, “Catadioptric camera calibration,” The 7th International Conference on Computer Vision, 1999, pp. 398-404.

[10]C. Geyer and K. Daniilidis, "Paracatadioptric camera calibration”, IEEE Transactions on Pattern Analysis and Machine Intelligence, Vol. 24, No. 5, pp. 687-695, 2002.
[11]J.P. Barreto and H. Araujo, "Issues on the geometry of central catadioptric image information,” IEEE Conference on Computer Vision and Pattern Recognition, 2001, pp. 422-427.

[12]J.P. Barreto and H. Araujo, "Geometric properties of central catadioptric line images,” The 7th European Conference on Computer Vision, 2002, pp. 237-251.

[13]X. Ying and H. Zha, "Simultaneously calibrating catadioptric camera and detecting line features using Hough transformation,” IEEE/RSJ International Conference on Intelligent Robots and Systems, 2005, pp. 1343-1348.

[14]C. Geyer and K. Daniilidis, "Catadioptric projective geometry," International Journal of Computer Vision, Vol. 45, No. 3, pp. 223-243, 2001.

[15]R. Hartley and A. Zisserman, Multiple view geometry in computer vision, Cambridge University Press, 2000.

[16]Y. Wu and Z. Hu, "A new constraint on the imaged absolute conic from aspect ratio,” Pattern Recognition Letters, Vol. 26, No. 8, pp. 1192-1199, 2005. 